УДК 534.1; 519.62

ISSN 1995-5499

DOI: https://doi.org/

Поступила в редакцию 29.10.2020

Подписана в печать 02.02.2021

\title{
ДИСКРЕТНАЯ МОДЕЛЬ СИНУС-ГОРДОНА С ГИСТЕРЕЗИСНЫМИ СВЯЗЯМИ
}

\author{
(C) 2020 А. В. Толкачев ${ }^{\varpi 1,2}$, П. А. Мелешенко ${ }^{1}$, А. В. Перова ${ }^{3}$ \\ ${ }^{1}$ Воронежский государственный университет \\ Университетская пл., 1, 394018 Воронеж, Российская Федерация \\ ${ }^{2}$ Воронежский государственный тесотехнический университет им. Г. Ф. Морозова \\ ул. Тимирязева, 8, 394613 Воронеж, Российская Федерация \\ ${ }^{3}$ Воронежский государственный технический университет \\ ул. 20-летия Октября, 84, 394006 Воронеж, Российская Федерация
}

\begin{abstract}
Аннотация. В статье исследуется коллективная динамика цепочки нелинейных маятников с гистерезисными связями между отдельными её элементами - дискретная гистерезисная модель синус-Гордона. Гистерезисные связи формализуются с помощью модели Боука - Вена, которая является удобными инструментом моделирования явления гистерезиса в механических системах. В работе представлены результаты моделирования эволюции локальных колебательных мод (бризеры) с помощью в интегрированной в MATLAB интерактивной среды Simulink. Используя фазовые портреты и спектры плотности мощности, отмечена регуляризирующая и фильтрующая роль гистерезисных элементов (в терминах параметров модели Боука - Вена). Анализ динамики, локализованных в цепочке собственных частот показывает, что в присутствии гистерезисных связей асимптотическое поведение соответствует предельному циклу. Рассматривается резонансные свойства системы синус-Гордона в случае силового гармонического воздействия на один из маятников в цепи. Моделируется бистабильный режим колебаний с помощью метода «сканирования» частоты. С его помощью рассчитывается амплитудно-частотная характеристика и определяется интервал частот, соответствующих неустойчивым режимам колебаний. Полученные результаты позволяют сделать выводы об эффективности гистерезисных блоков в качестве фильтрующих и регуляризирующих элементов рассматриваемой сложной колебательной системы.
\end{abstract}

Ключевые слова: нелинейные системы, гистерезис, модель Боука - Вена, солитон, бризер.

\section{ВВЕДЕНИЕ}

Колебательные процессы широко распространены в природе. В настоящее время указанная область исследований интенсивно развивается. Ежегодно различным аспектом теории колебаний посвящаются тысячи работ, в этой связи отметим наиболее близкие к изучаемым в настоящей статье системам работы [1-7]. При этом основными эталонными моделями теории колебаний являются такие объекты как линейный и нелинейный осцилляторы.

Толкачев Аким Владимирович e-mail: tolkachev.akim@mail.ru
Известно, что в механических системах в результате взаимодействиям большого числа колебательных элементов между собой наблюдется волновые явления [8]. Для исследования этого явления обычно используют модели конечных или бесконечных цепочек связанных (взаимодействующих) осцилляторов. Для непрерывных цепочек рассматривают соответствующие дифференциальные уравнения в частных производных. С прикладной точки зрения наиболее широкое распространение получили радиотехнические цепочки, которые используют как фильтры для выделения или подавления сигналов с частотами, лежащими в определенной полосе частот. Так Контент доступен под лицензией Creative Commons Attribution 4.0 License. The content is available under Creative Commons Attribution 4.0 License. 


\section{А. В. Толкачев, П. А. Мелешенко, А. В. Перова}

же отметим, что цепочки связанных осцилляторов являются наиболее широко используемой моделью сплошных сред [9].

Для описания различных физических явлений, как правило, используются линейные или квазилинейные модели. Такой подход является достаточно простым и наглядным, но не всегда адекватным методом моделирования современных физических и технических систем. В рамках линейной парадигмы происходит декомпозиция исходной системы на ряд простых подсистем, влияние которых на совокупную динамику может быть учтено изолировано. Однако в последнее время стало понятно, что допущение о линейности модели приводит к потере важных качественных характеристик исследуемых явлений.

Известно, что в случае нелинейной системы совокупность ее частей не определяет в полной мере особенности динамики и эволюции такой системы. Нелинейность проявляется в образовании новых структур, которые являются временно или пространственно когерентными. Эти новые структуры представляют собой достаточно большие объекты, обладающие уникальными свойствами. Одним из результативных выводов указанного подхода является концепция нелинейных волн - солитонов. Одной из базовых моделей для исследования таких нелинейных процессов является модель синус-Гордона (физическая реализация которой представляет собой цепочку нелинейных осцилляторов, связанных пружинами, работающими на кручение) [10-13].

Еще одним из примеров нелинейных физических явлений играющих значительную роль в современных исследованиях, является гистерезис. Математической теории систем с гистерезисом, трактует гистерезисные нелинейности как операторы или преобразователи с пространствами состояний. Наиболее полно конструктивные модели гистерезисных преобразователей, трактуемых как операторы, зависящие от своего начального состояния как от параметра и определенные на широком функциональном пространстве (например, на пространстве непрерывных функций или функций ограниченной вари- ации), изложены в монографии Красносельский М. А., Покровский А. В [14].

Математические модели таких сложных систем, как правило, одновременно включают дифференциальные уравнения и операторные соотношения между частью переменных; их исследование, в основном в случае простых гистерезисных операторов типа реле и люфтов, берет свое начало в классических работах теории управления и теории колебаний. В настоящее время свойства различных классов гистерезисных операторов достаточно хорошо изучены. К важным общим свойствам относится физическая реализуемость и коммутативность с монотонными преобразованиями времени: при изменении масштаба времени входа соответственно изменяется масштаб времени выхода. В то же время, вопросы, относящиеся к различным аспектам динамики систем с гистерезисом и, в том числе, различным динамическим режимам колебаний систем, не изучены достаточно полно. Их изучение осложняется еще и тем, что гистерезисные операторы не обладают свойством сильной дифференцируемости и могут иметь весьма сложные пространства состояний. К таким операторам относится, например, оператор Прейсаха, возникающий при моделировании систем с ферромагнитными элементами, а также в гидрологических моделях проникновения осадков в почву [15]. В последние годы, одной из наиболее часто используемых моделей гистерезиса является феноменологическая модель Боука - Вена. Эта модель формализуется посредством двух соотношений, - одного алгебраического и дифференциального уравнения. Модель Боука - Вена является удобным инструментом для формализации гистерезисных зависимостей, особенно в ситуации, когда гистерезисное звено является частью сложной системы [16-19].

Целью работы является дать анализ динамики солитона типа бризер в условиях гистерезисной связи, используя в качестве модели систему дифференциальных уравнений (дискретная гистерезисная модель синус-Гордона) и числено аппроксимировать решение задачи Коши, используя систему компьютерной алгебры. 


\section{1. МАТЕРИАЛЫ И МЕТОДЫ ИССЛЕДОВАНИЯ}

\section{1. Модель Боука - Вена}

В настоящей работе предлагается феноменологический подход к описанию гистерезисной нелинейности на основе модели Боука Вена). Для этого рассмотрим систему с одной степенью свободы как на рис. 1, тогда уравнение движения имеет вид

$$
\mu \ddot{u}(t)+F(u, z)=f(t),
$$

где $u(t)-$ смещение, $f(t)-$ внешняя сила, $F(u, z)$ - восстанавливающая сила, $\ddot{u}$ обозначает вторую производную по времени $t$, через $\mu$ обозначена масса.

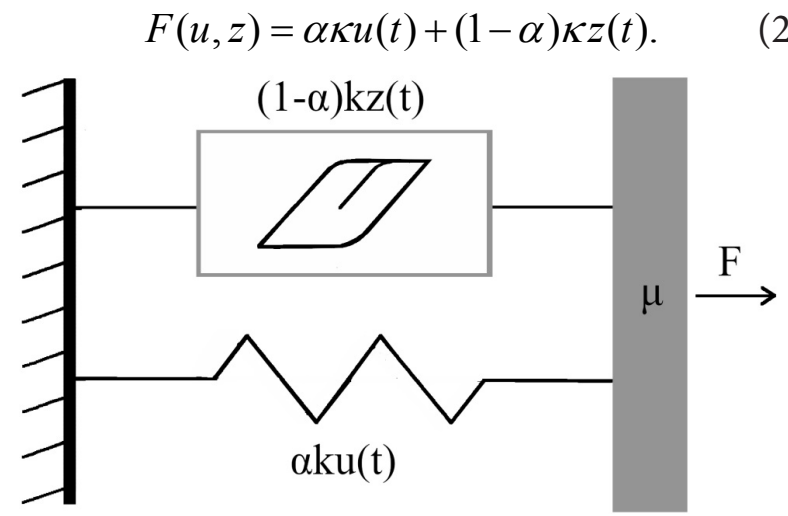

Рис. 1. Модель Боука - Вена

[Fig. 1. Bouc - Wen model]

Восстанавливающая сила $F(u, z)$ представляет собой суперпозицию из упругой $\alpha \kappa u(t)$ и гистерезисной составляющей $(1-\alpha) \kappa z(t)$, где $0<\alpha<1-$ параметр определяющий вклад в гистерезисную и упругую составляющую силы $F(u, z), \kappa-$ коэффициент жесткости. Гистерезисная часть включает в себя безразмерную переменную $z(t)$, которая является решением нелинейного дифференциального уравнения первого порядка.

$$
\dot{z}(t)=\left[A-|z(t)|^{\eta}(\beta+\operatorname{sign}(z(t) \cdot \dot{u}(t)) \gamma)\right] \cdot \dot{u}(t),(3)
$$

где $A, \beta$ и $\gamma-$ безразмерные параметры, которые определяют форму и размер петли гистерезиса, а $\eta \geq 1$ - скалярная величина, регулирующая гладкость перехода от упругого отклика к пластическому отклику. Отметим, что модель Боука - Вена способна в аналитическом виде описывать различные формы гисте- резисных циклов, которые отвечают поведению широкого класса гистерезисных систем.

\section{2. Система синус-Гордона с гистерезисными связям}

Наиболее известными и хорошо исследованными уравнениями математической физики являются уравнения, описывающие распространение волн в линейной среде. Для нелинейной среды, обладающей, к тому же, гистерезисными свойствами готовые методы решения таких уравнений отсутствуют.

Известно, что при распространении волн в нелинейных средах возможно появление солитонов - уединенных волн, ведущих себя подобно частицам. Одной из моделей, обладающей солитонным решением является система синус-Гордона - цепочка нелинейных маятников с упругими связями, работающими на кручение. Эта модель получила широкое распространение, как в области биологии, так и в физике.

Например, цепочку связанных маятников можно трактовать как простейшую модель многих нелинейных сред. Одним из примеров является задача о динамике дислокаций в кристаллах. Напомним, что дислокации линейные дефекты кристаллической решетки, нарушающие правильное чередование атомных плоскостей. Пластическая деформация кристаллов обычно связана с таким коллективным перемещением атомов, которое может быть описано, как «движение» дислокации, простейшая модель которых была предложена еще в 1930-х годах Френкелем Я. И. и Конторовой Т. А. [20]. Твердое тело рассматривается как совокупность слоев атомов, связанных упругими силами, роль которых играют пружины. Известно, что в процессе старения в различных материалах могут появляться дефекты, проявляющиеся, в том числе, посредством гистерезисных свойств. В этой связи представляется важным учет таких дефектов на модельном уровне - в рамках модели аналогичной модели синус-Гордона.

Ниже рассматривается механическая система с гистерезисными связями, физическая 


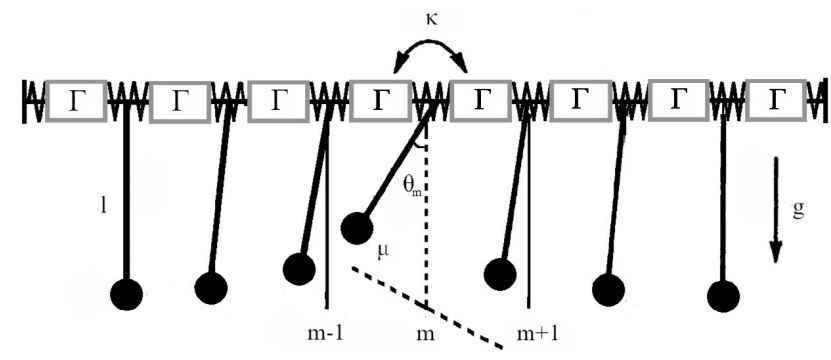

Рис. 2. Система синус-Гордона с гистерезисными связями

[Fig. 2. Sine-Gordon system with hysteretic links]

модель которой изображена на рис. 2. Она представляет собой цепочку одинаковых маятников, нанизанных на струну и связанных пружинами. Принципиальное отличие рассматриваемой механической системы состоит в том, что в связь между двумя соседними маятниками включена гистерезисная составляющая. Отметим, что указанная система представляет собой модификацию классической механической системы синус-Гордона.

Здесь и далее через $\mu$ обозначена масса маятника, тогда его момент инерции - $\mu l^{2}$, где $l-$ длина, а $\kappa-$ постоянная кручения пружины. Если отклонить $m$-й маятник от положения равновесия на угол $\theta_{m}\left(\theta_{m}(t)-\right.$ функция от времени $t$ ), то на него будет действовать момент силы тяжести $\mu g l \sin \theta_{m}$ и моменты силы кручения слева $\omega_{m}^{\text {left }}$ и справа $\omega_{m}^{r i g h t}$ соответственно. В этих предположениях уравнения движения для $m$ маятников можно представить в виде:

$$
\mu l^{2} \ddot{\theta}_{m}+\mu g l \sin \theta_{m}+\omega_{m}^{l e f t}+\omega_{m}^{r i g h t}=0 .
$$

Поделим слагаемые в уравнении (4) на величину $\mu l^{2}$ (избавимся от коэффициента при старшей производной $\ddot{\theta}$ ) и получим

$$
\ddot{\theta}_{m}+\frac{g}{l} \sin \theta_{m}+\frac{1}{\mu l^{2}} \omega_{m}^{\text {left }}+\frac{1}{\mu l^{2}} \omega_{m}^{\text {right }}=0 .
$$

Обозначим $\varpi=\sqrt{\frac{g}{l}}$, тогда

$$
\ddot{\theta}_{m}+\varpi^{2} \sin \theta_{m}+\frac{1}{\mu l^{2}} \omega_{m}^{\text {left }}+\frac{1}{\mu l^{2}} \omega_{m}^{\text {right }}=0 .
$$

Для упрощения записи будем считать, что масса $\mu$ и длинна $l$ маятника равна единицы, то есть $\mu=l=1$. Поскольку, в связи включена гистерезисная нелинейность, то соответствующие моменты $\omega_{m}^{\text {left }}(t)$ и $\omega_{m}^{\text {right }}(t)$ следует по- нимать как зависящие от времени $t$ выходы гистерезисного преобразователя Г[·]. Причем, входами для него будут

$$
\begin{aligned}
& y_{m}^{\text {left }}(t)=\kappa\left(\theta_{m}(t)-\theta_{m-1}(t)\right), \\
& y_{m}^{\text {right }}(t)=\kappa\left(\theta_{m}(t)-\theta_{m+1}(t)\right)
\end{aligned}
$$

соответственно. В рамках терминологии, применяемой в трактовке Красносельского-Покровского [12] для гистерезисных преобразователей, правый и левый моменты силы кручения имеют вид:

$$
\begin{aligned}
& \omega_{m}^{\text {left }}(t)=\Gamma\left[\omega_{m}^{\text {left }}\left(t_{0}\right), y_{m}^{\text {left }}\left(t_{0}\right)\right] y_{m}^{\text {left }}(t), \\
& \omega_{m}^{\text {right }}(t)=\Gamma\left[\omega_{m}^{\text {right }}\left(t_{0}\right), y_{m}^{\text {right }}\left(t_{0}\right)\right] y_{m}^{\text {right }}(t) .
\end{aligned}
$$

Отметим, что операторная трактовка гистерезисной нелинейности подразумевает негладкость соответствующего оператора, и, как следствие, значительно усложняет численный анализ рассматриваемой системы. Поэтому в дальнейшем для численного моделирования будет использована аппроксимация гистерезиса посредством модели Боука - Вена [1316]. В таком случае система синус-Гордона с гистерезисной нелинейностью примет вид:

$$
\begin{aligned}
\ddot{\theta}_{m}+\varpi^{2} \sin \theta_{m} & +\Omega_{m}^{\text {left }}\left(\theta_{m}-\theta_{m-1}, z_{m}^{\text {left }}\right)+ \\
& +\Omega_{m}^{\text {right }}\left(\theta_{m}-\theta_{m+1}, z_{m}^{\text {right }}\right)=0 .
\end{aligned}
$$

Для удобства введем следующие обозначения

$$
\begin{aligned}
& \Delta \theta_{m}^{\text {left }}=\theta_{m}(t)-\theta_{m-1}(t), \\
& \Delta \theta_{m}^{\text {right }}=\theta_{m}(t)-\theta_{m+1}(t),
\end{aligned}
$$

с учетом которых (7) примет

$$
\begin{aligned}
\ddot{\theta}_{m}+\varpi^{2} \sin \theta_{m}+ & \Omega_{m}^{\text {left }}\left(\Delta \theta_{m}^{\text {left }}, z_{m}^{\text {left }}\right)+ \\
& +\Omega_{m}^{\text {right }}\left(\Delta \theta_{m}^{\text {right }}, z_{m}^{\text {right }}\right)=0 .
\end{aligned}
$$

Где моменты силы кручения слева и справа: $\Omega_{m}^{\text {left }}\left(\Delta \theta_{m}^{\text {left }}, z_{m}^{\text {left }}\right)=\alpha \kappa\left(\theta_{m}-\theta_{m-1}\right)+(1-\alpha) \kappa z_{m}^{\text {left }}$, $\Omega_{m}^{r i g h t}\left(\Delta \theta_{m}^{r i g h t}, z_{m}^{r i g h t}\right)=\alpha \kappa\left(\theta_{m}-\theta_{m+1}\right)+(1-\alpha) \kappa z_{m}^{r i g .}$ а $z_{m}^{\text {left }}$ и $z_{m}^{\text {right }}-$ решения следующих нелинейных дифференциальных уравнений для $m$ маятников:

$$
\begin{aligned}
& \dot{z}_{m}^{\text {left }}=\left[\mathrm{A}-\left|z_{m}^{\text {left }}\right|^{\eta}\left(\beta+\operatorname{sign}\left(z_{m}^{\text {left }} \cdot \Delta \dot{\theta}_{m}^{\text {left }}\right)\right) \gamma\right] \cdot \Delta \dot{\theta}_{m}^{\text {left }} \\
& \dot{z}_{m}^{\text {right }}=\left[\mathrm{A}-\left|z_{m}^{\text {right }}\right|^{\eta}\left(\beta+\operatorname{sign}\left(z_{m}^{\text {right }} \cdot \Delta \dot{\theta}_{m}^{\text {right }}\right)\right) \gamma\right] \cdot \Delta \dot{\theta}_{m}^{\text {right }},
\end{aligned}
$$

где соответственно

$$
\Delta \dot{\theta}_{m}^{l e f t}=\dot{\theta}_{m}(t)-\dot{\theta}_{m-1}(t)
$$


Дискретная модель синус-Гордона с гистерезисными связями

$$
\Delta \dot{\theta}_{m}^{\text {right }}=\dot{\theta}_{m}(t)-\dot{\theta}_{m+1}(t) .
$$

Запишем в (8) упругие и гистерезисные составляющие, как ранее в (2). С учетом этого (8) перепишем

$$
\begin{aligned}
\ddot{\theta}_{m}+\varpi^{2} \sin \theta_{m} & +\alpha \kappa\left(\theta_{m}-\theta_{m-1}\right)+(1-\alpha) \kappa z_{m}^{\text {left }}+ \\
& +\alpha \kappa\left(\theta_{m}-\theta_{m+1}\right)+(1-\alpha) \kappa z_{m}^{r i g h t}=0 .
\end{aligned}
$$

Далее, перегруппируем слагаемые в левой части равенства

$$
\begin{aligned}
\ddot{\theta}_{m}+\varpi^{2} \sin \theta_{m}+ & \alpha \kappa\left(\theta_{m}-\theta_{m-1}\right)+\alpha \kappa\left(\theta_{m}-\theta_{m+1}\right)+ \\
& +(1-\alpha) \kappa z_{m}^{l e f t}+(1-\alpha) \kappa z_{m}^{r i g h t}=0 .
\end{aligned}
$$

И объединим 3 и 4 слагаемые

$$
\begin{aligned}
\alpha \kappa\left(\theta_{m}-\theta_{m-1}\right)+\alpha \kappa\left(\theta_{m}\right. & \left.-\theta_{m+1}\right)= \\
& =-\alpha \kappa\left(\theta_{m+1}-2 \theta_{m}+\theta_{m-1}\right) .
\end{aligned}
$$

Тогда сможем перезаписать (8) в виде

$$
\begin{aligned}
\ddot{\theta}_{m}+\varpi^{2} \sin \theta_{m}-\alpha \kappa\left(\theta_{m+1}-2 \theta_{m}+\theta_{m-1}\right)+ \\
+(1-\alpha) \kappa z_{m}^{\text {left }}+(1-\alpha) \kappa z_{m}^{\text {right }}=0 .
\end{aligned}
$$

В этих уравнениях $A, \beta, \gamma, \kappa, \eta, z_{m}^{\cdots}$ соответствуют ранее означенным параметрам модели Боука - Вена.

В системе уравнений (12) введен параметр $\alpha$, который задан на числовом промежутке $0<\alpha<1$, но для моделирования влияния гистерезиса в системе из $m$ маятников удобно рассматривать отрезок $0 \leq \alpha \leq 1$, так как позволяет варьировать значение гистерезисной составляющей в динамике волнового процесса. Если разрешить систему дифференциальных уравнений относительно старшей производной $\ddot{\theta}_{m}(t)=\frac{d^{2} \theta_{m}(t)}{d t^{2}}$ и раскрыть скобки в слагаемом $\alpha \kappa\left(\theta_{m+1}(t)-2 \theta_{m}(t)+\theta_{m-1}(t)\right)$ уравнений (12), при учете, что $\alpha=\kappa=1$, то получаем систему уравнений, которую называют дискретным синус-Гордон-уравнением (ДСГ-уравнением) [10].

$$
\begin{aligned}
\frac{d^{2} \theta_{m}(t)}{d t^{2}}=\varpi^{2} \sin \theta_{m}(t)+ & \\
& +\theta_{m+1}(t)-2 \theta_{m}(t)+\theta_{m-1}(t) .
\end{aligned}
$$

В настоявшее время такая система разностных уравнений хорошо изучена и имеет множество приложений. Например, для шунтовых переходов Джозефсона, решение системы типа бризер в этом случае ищут в виде [10].

$$
\theta_{m}(t)=4 \operatorname{arctg}(f(m, t)),
$$

где $f(m, t)=A c d(\Lambda t, k)$ в этом выражении $c d(\Lambda t, k)$ - эллиптическая функция Якоби с модулем $k=A^{4}$ и $\Lambda=\varpi /\left(1+A^{2}\right)$.

\section{РЕЗУЛЬТАТЫ И ИХ ОБСУЖДЕНИЕ}

Численное моделирование динамики рассматриваемой системы, описываемой уравнением (8) проводилось в интегрированной в MATLAB интерактивной среде Simulink. Для реализации указанной модели были определенны следующие параметры из табл.1.

Таблиия 1. Параметры для исследуемой системь

[Table 1. Parameters for the system]

\begin{tabular}{|c|c|}
\hline Параметр & 3начение \\
\hline$\mu$ & 1 \\
\hline$l$ & 1 \\
\hline$\kappa$ & 0.2 \\
\hline$g$ & 9.8 \\
\hline$\alpha$ & $0 \leq \alpha \leq 1$ \\
\hline$\beta$ & 0.05 \\
\hline$\gamma$ & 0.05 \\
\hline$\eta$ & 1 \\
\hline$A$ & 0.1 \\
\hline
\end{tabular}

Чтобы вычислить состояние модели во время симуляции использовался решатель ode14x (extrapolation). Этот решатель является комбинацией метода Ньютона и метода экстраполяции от текущего значения для того, чтобы посчитать состояние модели на следующем временном шаге. Таким образом, получим решение задачи Коши для системы (8) с модельным временем $t=1000$ и шагом дискретизации $h=0.01$. Предполагаем, что цепочка имеет конечную длину $m=100$ и подчиняется условиям периодичности $\left(\theta_{1}=\theta_{m+1}\right)$. Начальные условия $\theta_{1}\left(t_{0}\right), \dot{\theta}_{1}\left(t_{0}\right), \ldots, \theta_{m-1}\left(t_{0}\right)$, $\dot{\theta}_{m-1}\left(t_{0}\right), \theta_{m}\left(t_{0}\right), \dot{\theta}_{m}\left(t_{0}\right)$ для уравнений (8) генерируют множество решений, движущихся с различными скоростями по $(m, t)-$ плоскости. Для параметров гистерезисных блоков, формализуемых посредством модели Боука - Вена начальные условия по умолчанию нулевые $\left(z_{m}^{\text {right }}\left(t_{0}\right)=0, z_{m}^{\text {left }}\left(t_{0}\right)=0\right)$. 
Далее изучим, влияние гистерезисных связей в системе и рассмотрим случай, когда возбуждаются колебания у 50-го маятника с соответствующими для него начальными условиями $\left(\theta_{50}\left(t_{0}\right)=45, \dot{\theta}_{50}\left(t_{0}\right)=0\right)$. В таком случае в цепочке генерируется солитонное решение в виде дискретного бризера динамику которого можно увидеть на рис. 3. С точки зрения физической модели для данной цепочки мы отклонили 50-й маятник от положения равновесия на угол 45 градусов. В результате мы возбудили колебания, которые распространяются вдоль цепи. На рис. 3, 4 цветом варьируется значения амплитуд (в градусах) для каждого из компонент цепи.

На рис. 5, 6 представлены фазовые портреты для 50-го маятника в двух случаях, когда учитывается гистерезисная компонента в связях и не учитывается. Как следует из представленных результатов, в отсутствие гистерезисных связей $(\alpha=1)$ динамика маятников демонстрирует сложную колебательную структуру с различными режимами. Однако, в том случае, когда гистерезис в связях рассматривается колебания регуляризируются и имеет место предельный цикл.

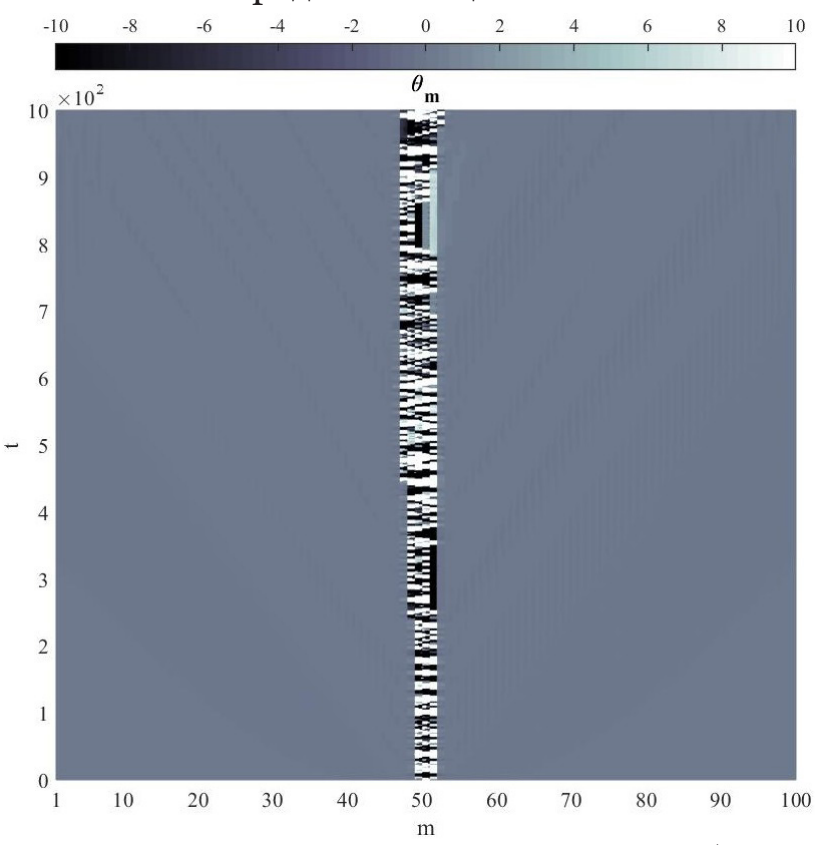

Рис. 3. Динамика локализованных колебаний (дискретный бризер) маятников в иепочке без гистерезиса

[Fig. 3. Dynamics of localized oscillations (discrete breather) of pendulums in a chain without hysteresis]

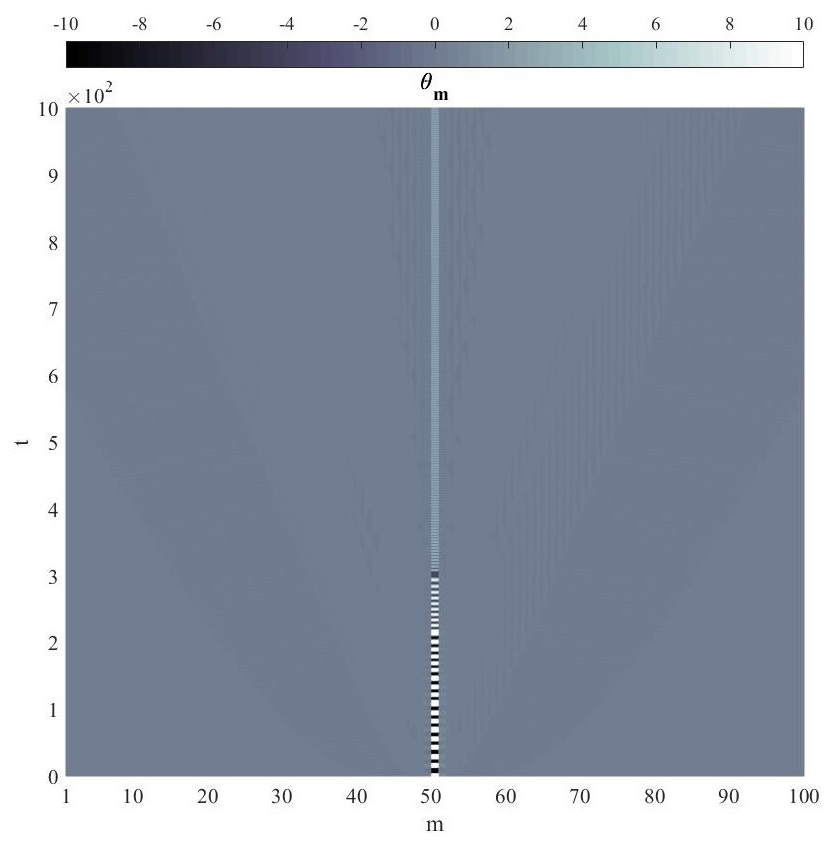

Рис. 4. Динамика локализованных колебаний (дискретный бризер) маятников в цепочке с гистерезисом

[Fig. 4. Dynamics of localized oscillations (discrete breather) of pendulums in a chain with hysteresis]

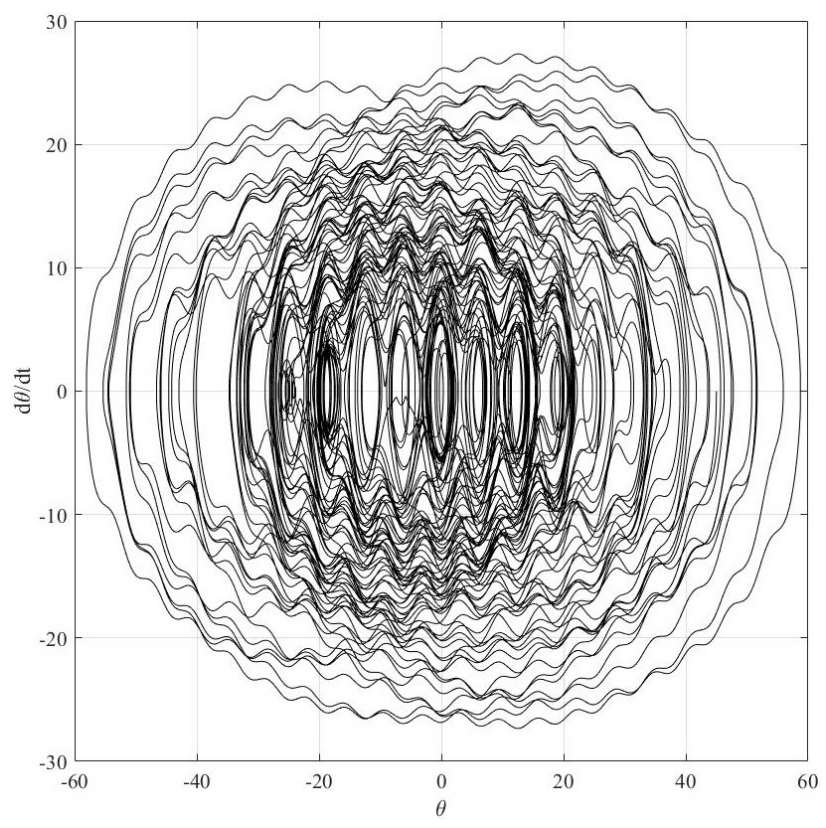

Рис. 5. Фазовый портрет 50-го маятника без гистерезиса в звеньях

[Fig. 5. Phase portrait of the 50th pendula without hysteresis in the links] 


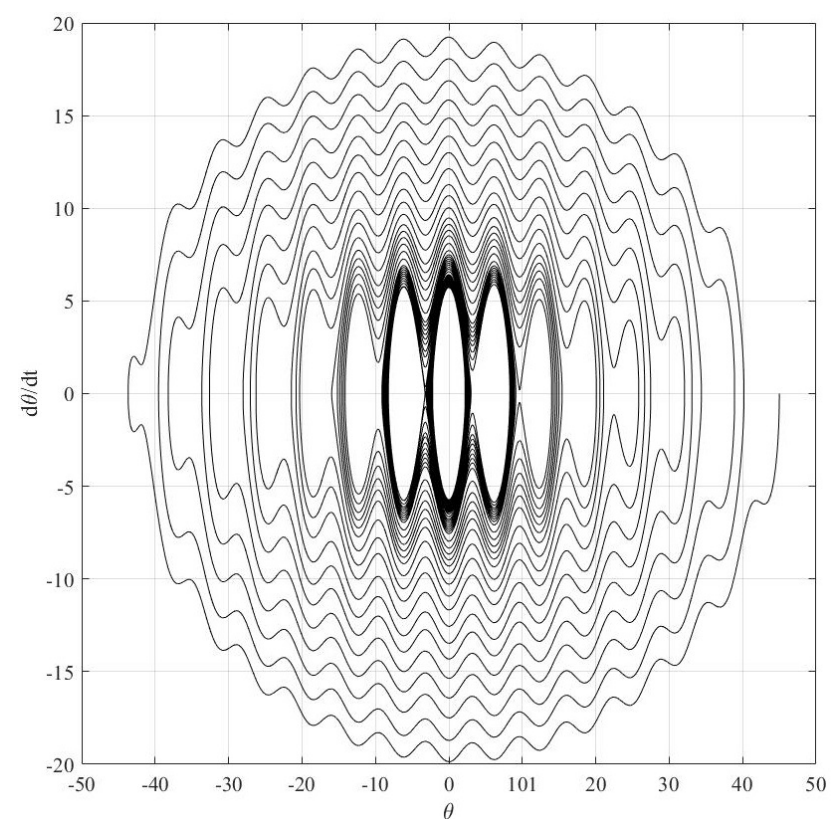

Рис. 6. Фазовый портрет 50-го маятника с гистерезисом в звеньях

[Fig. 6. Phase portrait of the 50th pendula with hysteresis in the links]

Рассмотрим влияние гистерезисных связей на динамику колебаний 50-го маятника посредством методов гармонического анализа. Такой анализ дает возможность оценить собственную частоту (моду) колебаний.

На рис. 7, 8 приведены спектральные характеристики колебательных движений с учетом гистерезиса $(\alpha=0.5)$ в связях и без него $(\alpha=1)$. А именно, применим алгоритм быстро преобразования Фурье для модельного сигнала, который получался в результате численного интегрирования системы (7) при $t=1000 \mathrm{c}, h=0.005$.

Как следует из рис. 7, 8, спектр плотности мощности для колебаний 50-го маятника модифицируется при учете гистерезисных компонент в связях, а именно, гистерезис в такой системе играет роль фильтра, который выделяет основную частоту (моду) и гасит остальные.

Особый интерес в прикладной науке вызывает исследование резонансных свойств колебательных систем. Рассмотрим случай, когда на 50-й маятник воздействует периодическая внешняя сила $F \sin (2 \pi v t)$, а так же предположим, что движения происходят в вязкой среде (возникает дополнительное слагаемое $\delta \dot{\theta}(t))$, тогда уравнение для 50-го в системе (8) примет вид:

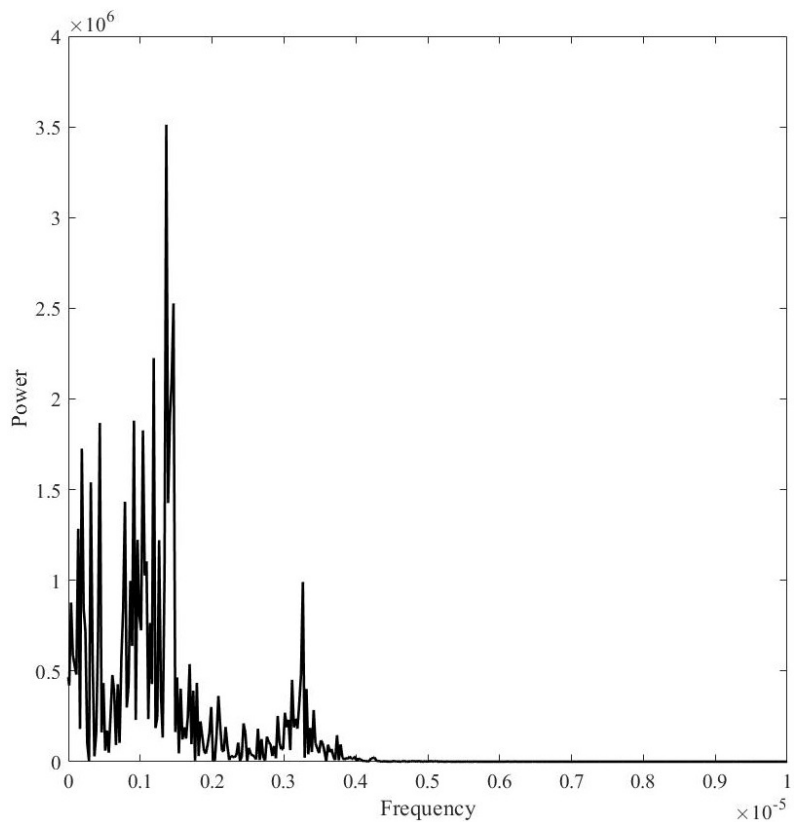

Рис. 7. Спектр плотности мощности для 50-го маятника без гистерезиса в звеньях

[Fig. 7. Power density spectrum for the 50th pendula without hysteresis]

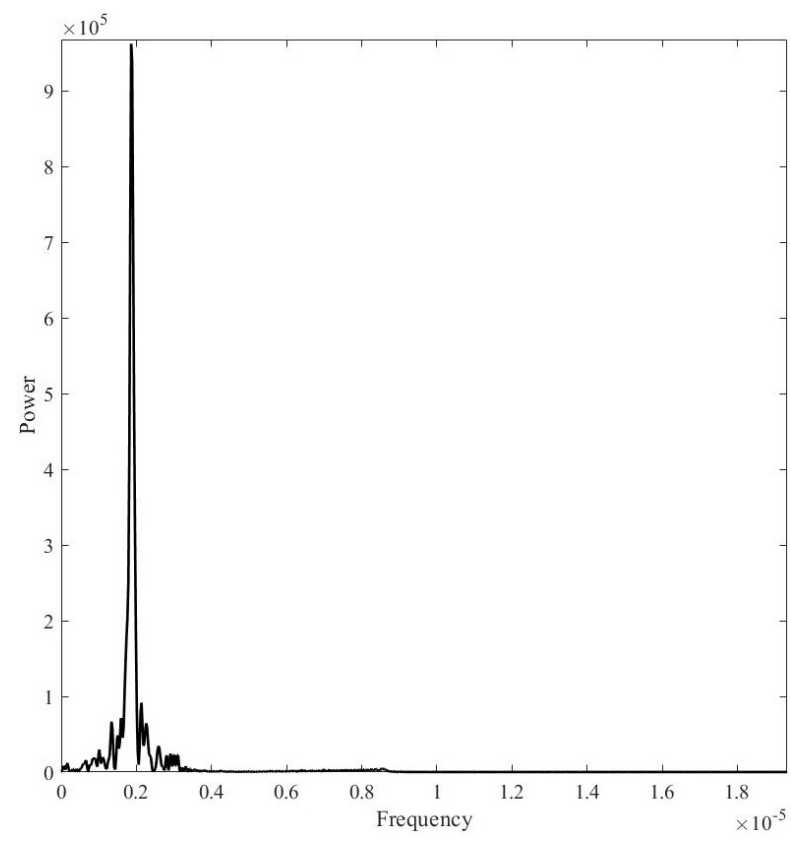

Рис. 8. Спектр плотности мощности для 50-го маятника с гистерезисом в звеньях

[Fig. 8. Power density spectrum for the 50th pendula with hysteresis]

$$
\begin{aligned}
& \ddot{\theta}_{50}+\varpi^{2} \sin \theta_{50}+\delta \dot{\theta}_{50}+\Omega_{50}^{\text {left }}\left(\Delta \theta_{50}^{\text {left }}, z_{50}^{\text {left }}\right)+ \\
&+\Omega_{50}^{\text {right }}\left(\Delta \theta_{50}^{\text {right }}, z_{50}^{\text {right }}\right)=F \sin (2 \pi v t)
\end{aligned}
$$

Далее, воспользуемся следующим способом изучения резонансных явлений. Он за- 


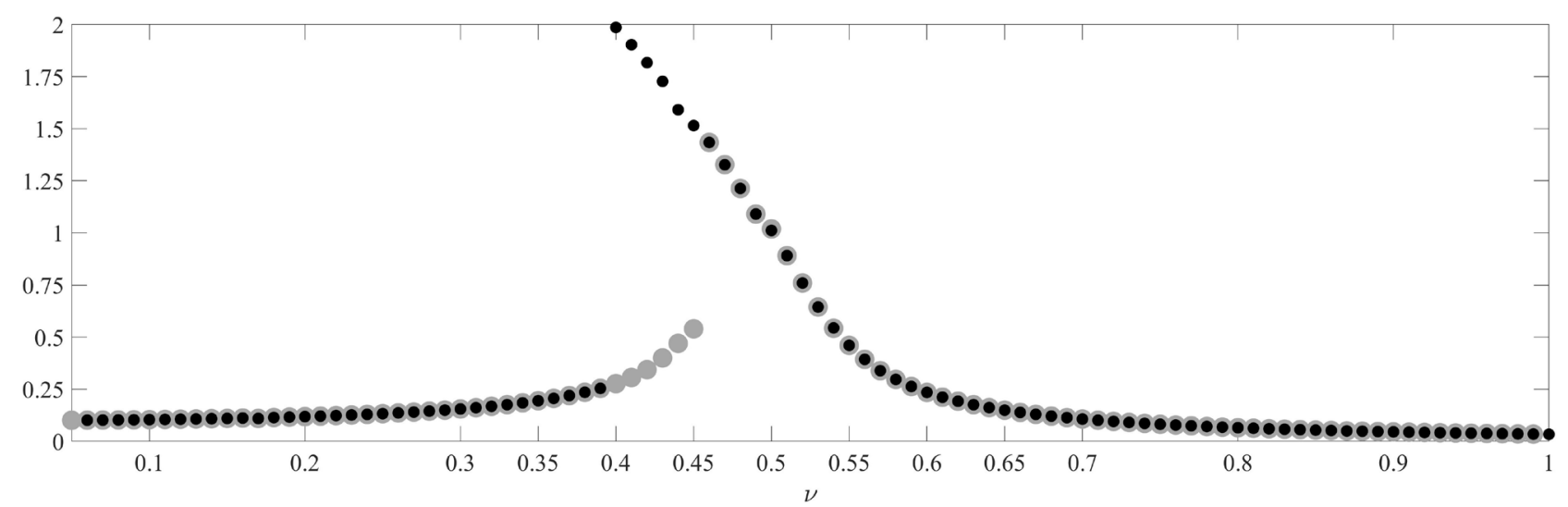

Рис. 9. Бистабильныий режим колебаний для 50-го маятника

[Fig. 9. Bistable oscillation mode for the 50th pendula]

ключается в медленном изменении частоты $v$ внешнего воздействия без прерывания процесса вынужденных колебаний («сканирование» частоты). В отличие от линейных колебательных систем, для которых каждому значению частоты соответствует определенная амплитуда, система из нелинейных маятников демонстрирует бистабильное поведение: когда при сканировании существует частота соответствующая двум разным режимам колебаний. В этом случае амплитуда установившихся колебаний изменяется скачкообразно, причем эти скачки происходят при различных значения частоты в зависимости от направления сканирования. На рис. 9 изображен бистабильный режим на отрезке $v \in[3.9,4.6]$ для 50-го маятника, полученный описанный ранее методом с шагом для частоты в 0.01. Где параметры в уравнении (15) будут теми же, что и ранее из табл. 1 , а новые $F=1$ и $\delta=0.01$.

\section{ЗАКЛЮЧЕНИЕ}

В настоящей работе исследована динамика колебательной системы со многими степенями свободы в условиях гистерезисной связи между отдельными звеньями. Рассматриваемая система может быть классифицирована как модифицированная механическая модель системы синус-Гордона в случае, когда связи между маятниками содержат гистерезисную нелинейность. Гистерезисная нелинейность формализовалась посредством модели Боука - Вена, допускающей достаточно простую численную реализацию решения системы дифференциальных уравнений, отвечающих моделируемой системы. На основе численного моделирования была исследована динамика бризера (локализованная колебательная мода) в указанной системе с учетом гистерезисных связей. Кроме того, результаты численного моделирования демонстрируют регуляризирующую роль гистерезисных связей в характере колебательных движений системы. Отмечены фильтрующие свойства гистерезисных связей с точки зрения гармонического анализа колебательных движений отдельных элементов. А также рассмотрен отклик системы на периодическое силовое воздействие для 50-го маятника (амплитудно-частотная характеристика).

\section{БЛАГОДАРНОСТИ}

Работа выполнена при финансовой поддержке РФФИ (проект 18-31-00394).

\section{КОНФЛИКТ ИНТЕРЕСОВ}

Авторы декларируют отсутствие явных и потенциальных конфликтов интересов, связанных с публикацией настоящей статьи.

\section{СПИСОК ЛИТЕРАТУРЫ}

1. Dynamics of Oscillator Chains / A. J. Lichtenberg [et al.] // Lect. Notes. Phys. - 2008. Vol. 728. - P. 21-121.

2. Torre, C. G. Linear Chain of Coupled Oscillators / C. G. Torre // Foundations of Wave Phenomena.- 2014. - Vol. 19. - P. 8-27. 
3. Sieber, J. Stability of a chain of phase oscillators / J. Sieber, T. Kalmar-Nagy // Phys. Rev. E. 2011. - Vol. 84. - P. 016227(1-6).

4. Coupled inverted pendulums: stabilization problem / M. E. Semenov [et al] // Arch. Appl. Mech. - 2017. - Vol. 87. DOI: https://doi. org/10.1007/s00419-017-1323-0

5. Неустойчивые колебания системы с гистерезисом: задачи стабилизации и управления / А. Л. Медведский [и др.] // Известия Российской академии наук. Теория и системы управления. - 2020. - № 4. - С. 58-82.

6. Динамика демпфирующего устройства на основе материала Ишлинского / М. Е. Семёнов [и др.] // Мехатроника, автоматизация, управление. - 2019. - Т. 20, № 2. - С. 106-113.

7. Гистерезисные преобразователи со случайными параметрами / С. В. Борзунов [и др.] // Математическое моделирование. 2019. - Т. 31, № 7. - С. 109-126.

8. Трубецков, Д. И. Линейные колебания и волны / Д. И. Трубецков, А. Г. Рожнев. - Москва : Издательство физико-математической литературы, 2001. - 419 с.

9. Тода, M. Теория нелинейных решеток / М. Тода. - Москва : Мир, 1984. - 262 с.

10. Филипов, А. Т. Многоликий солитон / А. Т. Филипов. - Москва : Наука, 1990. - 288 с.

11. Заславский, Г. М. Введение в нелинейную физику: от маятника до турбулентности и хаоса / Г. М. Заславский, Р. 3. Сагеев. - Москва: Наука, 1988. - 368 с.
12. Scott, A. C. A Nonlinear Kline - Gordon Equation / A. C. Scott // American Journal of Physics. - 1969. - Vol. 37. - P. 53-61.

13. Скотm, Э. Нелинейная наука. Рождение и развитие когерентных структур / Э. Скотт. Москва : ФИЗМАТЛИТ, 2007. - 560 с.

14. Красносельский, М. А. Системы с гистерезисом / М. А. Красносельский, А. В. Покровский. - Москва : Наука, 1983. - 272 с.

15. Modeling discontinuous flow through porous media using ODEs with Preisach operator / D. Flynn [et al.] // Physica B: Cond. Matter. 2008. - Vol. 403. - P. 440-442

16. Charalampakis, A. E. The response and dissipated energy of Bouc - Wen hysteretic model revisited / A. E. Charalampakis // Archve of Applied Mechanics. - 2015. - Vol. 85. - P. 1209-1223.

17. Ikhouane, F. On the Hysteretic Bouc Wen Model. Part I: Forced Limit Cycle Characterization / F. Ikhouane, J. Rodellar // Nonlinear Dynamics. - 2005. - Vol. 42. - P. 63-78.

18. Sine-Gordon system with hysteretic links / A. V. Tolkachev [et al.] // Journal of Physics: Conf. Series. - 2018. - Vol. 1096.

19. Discrete hysteretic sine-Gordon model: soliton versus hysteresis / P. A. Meleshenko [et al.] // MATEC Web of Conferences. - 2018. Vol. 241. - P. 01027.

20. Браун, О. М. Модель Френкеля - Конторовой. Концепция, методы, приложение / О. М. Браун, Ю. С. Кившарь. - Москва : ФИЗМАТЛИТ, 2008. - 536 с.

Толкачев Аким Владимирович - аспирант по направлению «Информатика и вычислительная техника», ассистент кафедры цифровых технологий Воронежского государственного университета и кафедры информатики и вычислительной техники Воронежского государственного лесотехнического университета им. Г. Ф. Морозова.

E-mail:tolkachev.akim@mail.ru

ORCID iD: https://orcid.org/0000-0002-4968-5253

Мелешенко Петр Александрович - канд. физ.-мат. наук, доцент кафедры цифровых технологий Воронежского государственного университета.

E-mail: melechp@yandex.ru

ORCID iD: https://orcid.org/0000-0003-3619-7258

Перова Алла Владимировна - канд. техн. наук, доцент кафедры технологии машиностроения Воронежского государственного технического университета.

E-mail: pva7@mail.ru

ORCID iD: https://orcid.org/0000-0003-21-381799 
DOI: https://doi.org/

Received 29.10.2020

ISSN 1995-5499

Accepted 02.02.2021

\title{
DISCRETE SINE-GORDON MODEL WITH HYSTERETIC LINKS
}

\author{
(c) 2020 A. V. Tolkachev ${ }^{\bowtie 1,2}$, P. A. Meleshenko ${ }^{1}$, A. V. Perova ${ }^{3}$ \\ ${ }^{1}$ Voronezh State University \\ 1, University Square, 394018 Voronezh, Russian Federation \\ ${ }^{2}$ Voronezh State University of Forestry and Technologies Named After G. F. Morozov \\ 8, Timiryazeva Street, 394613 Voronezh, Russian Federation \\ ${ }^{3}$ Voronezh State Technical University \\ 84, 20 let Oktyabrya Street, 394006 Voronezh, Russian Federation
}

\begin{abstract}
Annotation. The article considers the collective dynamics of a set of nonlinear pendulums with hysteresis coupling between individual elements - the so-called discrete hysteretic sine-Gordon model. Hysteresis couplings can be formalized using the Bouc - Wen model, which is a convenient tool for modelling the phenomenon of hysteresis in mechanical systems. The article presents the simulation results for the evolution of localized oscillatory modes (breathers) obtained using the interactive Simulink environment integrated with MATLAB. Phase portraits and power spectrum density demonstrated the regularisation and filtering role of the hysteresis elements (using the terms of the Bouc - Wen model). Analysis of the dynamics of the localized natural frequencies showed that in the presence of hysteresis coupling, the asymptotic behaviour corresponds to the limiting cycle. We also analysed the resonance properties of the discrete hysteresis sine-Gordon system in the case of harmonic excitation of one of the pendulums. A bistable oscillatory mode was simulated using the frequency "scan" method. The method was used to calculate the amplitude-frequency characteristic and determine the frequency interval corresponding to unstable oscillatory modes. The obtained results demonstrated the efficiency of the hysteresis blocks as filtering and regulating elements of the complex nonlinear oscillatory system considered in the study.
\end{abstract}

Keywords: nonlinear system, hysteresis, Bouc - Wen model, solution, breathers.

\section{CONFLICT OF INTEREST}

The authors declare the absence of obvious and potential conflicts of interest related to the publication of this article.

\section{REFERENCES}

1. Lichtenberg A. J., Livi R, Pettini M., Ruffo S. Dynamics of Oscillator Chains. Lect. Notes. Phys. 2008. V. 728. P. 21-121.

2. Torre C. G. Linear Chain of Coupled Oscillators. Foundations of Wave Phenomena. 2014. V. 19. P. 8-27.

3. Sieber J., Kalmar-Nagy T. Stability of a chain of phase oscillators. Phys. Rev. E. 2011. V. 84. P. 016227(1-6).

Tolkachev Akim V. e-mail: tolkachev.akim@mail.ru
4. Semenov M. E., Solovyov A. M., Popov M. A., Meleshenko P. A. Coupled inverted pendulums: stabilization problem. Arch. Appl. Mech. 2017. V. 87. DOI: https://doi.org/10.1007/s00419-017-1323-0.

5. Medvedsky A. L., Meleshenko P. A., Nesterov V. A., Reshetova O. O., Semenov M. E., Solovyov A. M. Unstable Oscillating Systems with Hysteresis: Problems of Stabilization and Control. Herald of the Russian Academy of Sciences. Control theory and systems. 2020. V. 4 P. 58-82.

6. Semenov M. E., Matveev M. G., Meleshenko P. A., Meleshenko P. A., Solovyov A. M. Dynamics of a damping device based on Ishlinsky's material. Mekhatronika, Avtomatizatsiya, Upravlenie. 2019. V. 20. .№. 2. P. 106-113.

7. Borzunov S. V., Semenov M. E., Sel'vesyuk N. I., Meleshenko P. A. Hysteresis converters with random parameters. Matem. Mod. 2019. V. 31. No 7. P. 109-126. 
8. Trubetskov D. I. Rozhnev A. G. Linear oscillations and waves. Moscow: Publishing house of physical and mathematical literature. 2001.419 p.

9. Toda M. Theory of Nonlinear Lattices. Berlin : Springer, 1981. $204 \mathrm{p}$.

10. Filippov A. T. Many-Sided soliton. Moscow: Nauka. 1990. 288 p.

11. Zaslavsky G. M, Sagdeev R. Z. Nonlinear Physics: From the Pendulum to Turbulence and Chaos. Moscow : Nauka, 1988. 368 p.

12. Scott A. C. Emergence and Dynamics of Coherent Structures. Moscow : FIZMATLIT, 2007. $560 \mathrm{p}$.

13. Scott A. C. A Nonlinear Kline - Gordon Equation. American Journal of Physics. 1969. V. 37. P. 53-61.

14. Krasnoselsky M. A., Pokrovsky A. V. Systems with hysteresis. Moscow : Nauka, 1983. $272 \mathrm{p}$.

15. Flynn D. Zhezherun A., Pokrovskii A., O'Kane J. P. Modeling discontinuous flow through porous media using ODEs with Preisach operator. Physica B: Cond. Matter. 2008. V. 403. P. $440-442$.
16. Charalampakis A. E. The response and dissipated energy of Bouc - Wen hysteretic model revisited. Archve of Applied Mechanics. 2015. V. 85. P. 1209-1223.

17. Ikhouane F., Rodellar J. On the Hysteretic Bouc - Wen Model. Part I: Forced Limit Cycle Characterization. Nonlinear Dynamics. 2005. V. 42. P. 63-78.

18. Tolkachev A. V., Semenov M. E., Meleshenko P. A., Reshetova O. O., Klinskikh A. F., Karpov E. A. Sine-Gordon system with hysteretic Journal of Physics: Conf. Series. 2018. V. 1096.

19. Meleshenko P. A., Tolkachev A. V., Semenov M. E., Perova A. V., Barsukov A. I., Klinskikh A.F. Discrete hysteretic sine-Gordon model: soliton versus hysteresis. MATEC Web of Conferences. 2018. V. 241.

20. Braun O. M., Kivshar Y. The Frenkel Kontorova Model: Concepts, Methods, and Applications. Berlin : Springer, 2004.

Tolkachev Akim V. - postgraudate student, lecturer, Department of Digital Technologies, Voronezh State University, Department of Informatics and Computer Science Voronezh State University of Forestry and Technologies Named after G.F. Morozov.

E-mail: tolkachev.akim@mail.ru

ORCID iD: https://orcid.org/0000-0002-4968-5253

Meleshenko Petr A. - PhD in Physics and Mathematics, Associate Professor, Department of Digital Technologies, Voronezh State University.

E-mail: melechp@yandex.ru

ORCID iD: https://orcid.org/0000-0003-3619-7258

Perova Alla V. - PhD in Technical Sciences, Associate Professor, Department of Mechanical Engineering Technology Voronezh State Technical University.

E-mail: pva7@mail.ru

ORCID iD: https://orcid.org/0000-0003-2199-3817 